\title{
Sex-linked molecular markers for two cold-hardy kiwifruit species, Actinidia arguta and A. kolomikta
}

\author{
I. Hale, A.T.O. Melo and H. Gustafson \\ University of New Hampshire, College of Life Science and Agriculture, Department of Agriculture, Nutrition and Food \\ Systems, Durham, NH, USA
}

\begin{abstract}
Summary
Developing improved fruiting varieties of kiwiberry (Actinidia arguta) and other species of cold-hardy kiwifruit (e.g., A. kolomikta) is highly resource-intensive due to the dioecy, extended juvenile phase (3-5 years), and vegetative vigor of these woody, vining species. The ability to identify gynoecious plants at the seedling stage is therefore desired as a means of increasing the resource-use efficiency of breeding programs. In this study, sex-linked polymorphisms in both species were identified through reference-independent genotyping-by-sequencing (GBS) analysis; validated in independent populations; and converted into cost-effective, gel-based PCR markers appropriate for marker-assisted breeding programs. The marker for A. arguta, designed using male-allele-specific primers based on a SNP within a 157 bp GBS amplicon, exhibits perfect sex-linkage across a comprehensive set of kiwiberry germplasm currently available through the US nursery trade and the USDA's National Plant Germplasm System (NPGS). By multiplexing conserved primers for a co-located internal 81 bp control amplicon, this male-specific marker was made effectively co-dominant, thereby enhancing scoring accuracy. Similarly, the $161 \mathrm{bp}$ marker for A. kolomikta was designed with male-allele-specific primers based on a 3 bp indel within a 270 bp GBS fragment and exhibits perfect sex-linkage throughout the available $A$. kolomikta germplasm in North America. Both markers were validated in independent populations and are now being used for high-throughput sex-screening assays of seedling populations.
\end{abstract}

Keywords

kiwiberry, cold-hardy kiwifruit, Actinidia arguta, Actinidia kolomikta, marker-assisted selection, sex screening

\section{Introduction}

Although the genus Actinidia is comprised of an estimated 76 species (Ferguson and Huang, 2007) native to a wide range of habitats across eastern Asia, nearly all of the $>$ US\$ 2 billion global kiwifruit trade (FAO, 2013) is based on cultivars of two closely related and recently domesticated temperate species: A. chinensis var. deliciosa and, increasingly, A. chinensis var. chinensis (Ferguson, 2013). While cold-hardier members of the genus, namely A. arguta and A. kolomik$t a$, have long been recognized for their economic potential, particularly at higher elevations and in more northern latitudes (Goodale, 1981), these species have received relatively

\section{Significance of this study}

What is already known on this subject?

- Like all kiwifruit species within the diverse Actinidia genus, the cold-hardy species $A$. arguta and A. kolomikta are dioecious. Given the long juvenile periods of these woody, perennial lianas, dioecy presents a significant and fundamental inefficiency to breeding programs, one that could be solved through the use of sex-linked molecular markers to identify male and female plants at the seedling stage.

What are the new findings?

- Using diverse association mapping panels and independent validation populations, this study develops, validates, and reports the full details of a low-cost, sex-linked molecular marker for each species. By providing the first publically-available molecular markers specifically developed for $A$. arguta and $A$. kolomikta, this study paves the way for the marker-assisted improvement of these species.

What is the expected impact on horticulture?

- By adopting the use of these markers, marker-assisted breeding programs for these emerging horticultural crop species can greatly improve their resource efficiency, thereby accelerating the development of improved cultivars.

little attention by formal breeding programs in the United States since their initial introduction into the country as ornamentals in the 1870's (Ferguson and Seal, 2008). On one hand, this lack of investment is surprising, given the growing number of examples of the commercial viability of extant selections and the numerous desirable traits of their small, grape-sized fruits, including impressive nutritional profiles (Nishiyama et al., 2004; Crowhurst et al., 2008; Huang et al., 2013), exceptional flavor (Ferguson, 2013), and hairless, edible skins. On the other hand, systematic plant breeding programs are long-term, high-investment propositions under the best of conditions; and the risks are inherently higher for a novel, resource-intensive crop with essentially no current commercial base. Not only do these vigorous, woody lianas require substantial physical infrastructure (e.g., trellising), intensive vine management (i.e., training and multiple prunings per year), and significant physical space $\left(>14 \mathrm{~m}^{2}\right.$ per plant under commercial production systems); they also require up to five years or more before reaching reproductive maturity.

Complicating matters further is the fact that, although most angiosperms are hermaphroditic (Friis et al., 1987; 
Renner and Ricklefs, 1995), all Actinidia species are reported to be functionally dioecious (Seal and McNeilage, 1988). This trait presents a fundamental inefficiency to breeding programs, particularly in light of the $\sim 60-70 \%$ male bias routinely observed in randomly segregating populations (R. Guthrie and D. Jackson, pers. commun.). With neither sexually dimorphic traits at the seedling stage nor cytologically-evident sex chromosomes within Actinidia spp. (Ming et al., 2011), the sex of any given kiwifruit vine is discernible only once it reaches reproductive maturity. Sex-linked molecular markers developed in other dioecious genera [e.g., Asparagus (Reamon-Buettner and Jung, 2000), Carica (Deputy et al., 2002), and Cannabis (Mandolino et al., 1999)] demonstrate the potential of enhancing breeding efficiency by allowing an effective increase in population sizes, given resource constraints. Similarly in A. chinensis var. chinensis, a sub-telomeric sex-associated locus was discovered with tightly linked male (SMY) and female (SMX) associated loci (Fraser et al., 2009), though the causative genes have yet to be identified and the markers unfortunately fail to exhibit sex-linkage in either cold-hardy species, $A$. arguta or A. kolomikta.

While the specific mechanisms underlying sex determination in Actinidia spp. remain unknown, it has long been observed that male plants within the genus possess staminate flowers with rudimentary ovaries while female plants appear hermaphroditic but are male-sterile (Ferguson, 2013). Due to the apparent disomic segregation of ovary abortion $(0)$ and pollen fertility (P) across various ploidy levels (Testolin et al., 1999), these two traits have been hypothesized to be controlled by two tightly-linked genes, with op/OP individuals developing as male and op/op individuals as female (Ainsworth, 1999). This genetic model finds support in the rare occurrences of both sterile (putative $o p / O p$ ) and hermaphroditic (putative op/oP) individuals (Ferguson, 2013), as well as in the observed linkage between the sex-associated SMX and SMY regions identified in A. chinensis var. chinensis (Fraser et al., 2009).

As no marker-assisted selection protocols are currently available to support public breeding efforts in the relatively understudied species $A$. arguta and A. kolomikta, this investigation was undertaken to develop cost-effective, PCR-based assays for sex-linked loci in both species. In light of the recent ban on importing additional Actinidia germplasm into the US due to concerns over Pseudomonas syringae pv. actinidiae (USDA, 2010), the target germplasm for this study consists of all available accessions in the US nursery trade and the USDA's National Plant Germplasm System (NPGS) (see Melo et al., 2017).

\section{Materials and methods}

\section{Plant material}

For the initial genotyping-by-sequencing (GBS) association analysis, leaf tissue was sampled from the complete set of non-redundant accessions of the North American collection of cold-hardy kiwifruit germplasm, as described by Melo et al. (2017). Specifically, this set included 60 accessions of $A$. arguta (42 females, 17 males, 1 unknown) and 13 accessions of $A$. kolomikta (10 females, 3 males) (see Supplemental information - Table S1). For subsequent marker validation, the following three independent populations were used: 1) An open-pollinated population of 17 mature, half-sib $A$. arguta lines (10 females and 7 males; mother: A. arguta cv. Ogden Point) from the University of Minnesota
Landscape Arboretum Horticultural Research Center (HRC); 2) An open-pollinated population of 21 mature, half-sib A. arguta lines (11 females and 10 males; mother: A. arguta cv. Hortgem Tahi) from Kiwi Berry Organics Co. in Danville, PA; and 3) An open-pollinated population of 21 mature, halfsib A. kolomikta lines (10 females and 11 males; mother: A. kolomikta cv. Nahodka) from the HRC.

\section{DNA isolation, library preparation, and sequencing}

Genomic DNA was extracted using a modified CTAB protocol. Specifically, $100 \mathrm{mg}$ of fresh, healthy leaf tissue was lyophilized and ground to a powder in a Retsch mill, using three 3-mm tungsten carbide beads per $1.1 \mathrm{ml}$ tube. The resultant powder was incubated at $65^{\circ} \mathrm{C}$ in $750 \mu \mathrm{l}$ of DNA extraction buffer (CTAB, $1 \%$ polyvinylpyrritate and $2 \%$ $2 \beta$-mercaptoethanol) for 45 minutes, with occasional mixing. After adding $750 \mu \mathrm{l}$ of chloroform:isoamyl alcohol (24:1 $\mathrm{v} / \mathrm{v}$ ) and mixing by gentle inversion, tubes were centrifuged at $10,000 \mathrm{rpm}$ for 10 minutes and the aqueous phase recovered. $10 \%$ volume of $65^{\circ} \mathrm{C} \mathrm{CTAB} / \mathrm{NaCl}$ solution was added, followed by a second chloroform:isoamyl alcohol step. $\mathrm{Nu}-$ cleic acids were precipitated with an equal volume of $95 \%$ isopropanol and centrifuged at 13,000 rpm for 10 minutes. The recovered pellet was washed twice with $700 \mu \mathrm{l}$ of $70 \%$ ethanol, air dried, and resuspended in $50 \mu \mathrm{l}$ of $10 \mathrm{mM}$ Tris. Prior to GBS library preparation, the isolated gDNA was purified using Zymo DCC-10 clean-up columns (Zymo \#D4011).

Multiplexed GBS libraries were prepared according to the two enzyme (PstI - MspI) protocol described by Poland et al. (2012). Using a set of $6-10$ bp barcodes from that protocol, the 60 accessions from $A$. arguta and 13 accessions from A. kolomikta were multiplexed and sequenced on an Illumina HiSeq 2500 at the Hubbard Center for Genome Studies, University of New Hampshire. FASTQ files of the 150 bp pairedend (PE) sequence data were generated using CASAVA 1.8.3; and all parsed, high-quality, PE reads were deposited in the NCBI Sequence Read Archive. Detailed information about the 73 accessions in this study, such as genotype names, NPGS accession numbers, reported and observed gender information, the number of high quality PE reads, the number of variants called for each genotype, the NCBI SRA submission IDs, etc., can be found in Supplemental information - Table S1.

\section{Genotyping}

The CASAVA-processed, raw sequence data from both species were submitted for analysis to version 2.1 (SNP + indel functionality) of the GBS-SNP-Calling Reference Optional Pipeline (GBS-SNP-CROP; Melo et al., 2016). As discussed in more detail by Melo et al. (2016), the pipeline calls Trimmomatic (Bolger et al., 2014) to trim reads based on a sequence of three contiguous nucleotides with an average Phred score $\mathrm{Q} \leq 30$. High-quality reads at least $32 \mathrm{bp}$ in length were then demultiplexed into read pairs, by genotype. For this study, in an attempt to avoid dominant tags present only in male genotypes, the most read-abundant female genotype from each species (cv. ORUS 2-16 for A. arguta and cv. Matonaya for A. kolomikta) was chosen to build a reduced-representation reference (i.e., Mock Reference; see Melo et al., 2016) to facilitate GBS read mapping and variant (SNP and indel) discovery. After alignment to the mock reference using BWAmen (Li and Durbin, 2009), SAMtools (Li et al., 2009) was used to retain only those read pairs that mapped in proper orientation with no supplementary or duplicate alignments for variant calling. As the two species evaluated have different ploidy levels (Melo et al., 2017), we used the respective, 
recommended GBS-SNP-CROP parameters for step 7 to call variants for diploid (A. kolomikta) and tetraploid (A. arguta) species.

A simple search for SNP and indel markers exhibiting homozygosity for all female accessions and heterozygosity for $>95 \%$ of the male accessions yielded sets of candidate sex-associated markers for A. arguta and A. kolomikta. The relaxed (>95\%) heterozygosity requirement for male accessions was chosen due to the fact that GBS, by virtue of its non-uniform read depth across all loci, can incorrectly score heterozygotes as homozygotes (see Melo et al., 2016). For each candidate marker, the associated FASTA entry from the Mock Reference (i.e., the consensus GBS fragment or centroid) was examined to understand the sequence context of the putative male/female polymorphism and guide PCR-based primer design for confirmation within the original population and validation in independent populations.

\section{PCR marker development and validation}

Primers for polymorphic amplicons putatively associated with sex were designed using Primer3 (Rozen and Skaletsky, 2000) and initially tested on a small sample of accessions of known gender from the same population used for GBS analysis (see Supplemental information - Table S1). All PCRs were conducted in a total reaction volume of $20 \mu \mathrm{l}(0.25 \mathrm{mM}$ of each primer, $100 \mu \mathrm{M}$ of each dNTP, $0.75 \mathrm{U}$ Taq DNA Polymerase, 10× standard Taq buffer [NEB N0273], and 50-100 ng of template DNA). Cycling conditions consisted of $5 \mathrm{~min}$ at $94^{\circ} \mathrm{C} ; 32$ cycles of $30 \mathrm{~s}$ at $94^{\circ} \mathrm{C}, 30 \mathrm{~s}$ at the appropriate primer annealing temperature, and $15 \mathrm{~s}$ at $68^{\circ} \mathrm{C}$; followed by a final 5-minute elongation step. Amplified products were separated at $75 \mathrm{~V}$ on a $2 \% \mathrm{TBE} / \mathrm{EtBr}$ agarose gel for $60 \mathrm{~min}$ and imaged with UV transillumination. Following successful amplification on the initial sample of accessions, promising markers were used to screen the entire US kiwiberry collection, as well as the independent segregating populations.

\section{Results}

\section{Bioinformatics analysis and primer design}

Using only the single most read-abundant genotype to build each species-specific Mock Reference, the GBS-SNPCROP pipeline (v2.1) called a total of 32,222 (mean depth $\mathrm{D}=$ $60.24)$ and $12,486(\mathrm{D}=57.06)$ variants for $A$. arguta (60 accessions) and $A$. kolomikta (13 accessions), respectively. For A. arguta, of the total variants called, 30,468 were SNPs and 1,754 were indels, while for $A$. kolomikta the pipeline called 12,067 SNPs and 419 indels. These sets of variants were called using 286.3 and 69.6 million PE high quality reads for A. arguta and A. kolomikta, respectively (Table 1). The two species exhibited similar values of overall loci heterozygosity (Hetero), homozygosity (Homo), and missing data (NA), with the intraspecific averages being $27.7 \%$ (Hetero), $60.4 \%$ (Homo), and $11.9 \%$ (NA) for A. arguta and $35.3 \%$ (Hetero), $58.0 \%$ (Homo), and 6.7\% (NA) for A. kolomikta (Table 1).

The initial association analyses yielded three candidate sex-linked clusters in A. arguta and four in A. kolomikta [hereafter noted with the prefixes aC (arguta Cluster) and kC (kolomikta Cluster), respectively] (Tables 1 and 2), several of which contained multiple polymorphisms (for full details, see Supplemental information - File S1). Sex-linked sequences were prioritized for marker development based on practical constraints of primer design as well general considerations of efficiency, reproducibility, and ease of scoring in the lab. In general, allele-specific primers were pre- ferred over CAPS markers, as we wished to avoid the extra cost (time and money) of restriction digestion. Additionally, length polymorphisms were preferred over SNPs due to their relative ease of scoring on gels, and the molecular weights of amplicons were prioritized with regard to downstream gel resolution. Three putative sex-linked polymorphic sequences could not be immediately developed into PCR markers due to variant locations within centroids that restricted primer design (clusters aC70626, kC693, and kC1273). The majority of putative sex-linked variants, however, were located more than $20 \mathrm{bp}$ from either end of a centroid, thereby permitting the placement of primers on both sides of the variant (Table 2).

Experimental primer pairs were designed for variants within two clusters in A. arguta [aC36306 (1 SNP) and aC123485 (1 indel)] and two clusters in A. kolomikta [kC48400 (2 SNPs) and kC72369 (1 indel); see Table 2]. For variants involving no length polymorphism (e.g., SNPs), primer pairs were designed to amplify only male-specific sequences in order to avoid a second, costly restriction digestion step (i.e., CAPS marker). To accomplish such male-specific amplification, one of the primers (either forward or reverse) was designed such that the final nucleotide on the 3' end matched only the male variant and the antepenultimate nucleotide on the 3' end mismatched both male and female variants, effectively blocking extension of female variants. Two candidate variants were indels and required no such designed mis-match. In cluster aC123485, a co-dominant primer pair was designed around a $10 \mathrm{bp}$ indel, yielding two amplicons in males and one in females, whereas in cluster

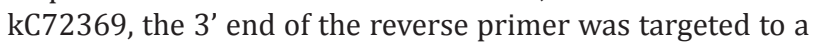
3 bp male insertion to create a male-dominant marker.

\section{Marker validation and loci characteristics}

Primer pairs were first tested on a small set of male and female accessions of the corresponding species (see Supplemental information - Table S1) to confirm the verity of the GBS analysis prior to confirmation across the larger population. Following this initial confirmation, primer pairs designed within clusters aC36306, aC123485, and kC72369 were tested across the remaining accessions of known gender in the original, training populations (Supplemental information - Table S1). Primers for cluster kC48400 failed to amplify in accordance with the GBS results; so this cluster was dropped from further investigation. Both A. arguta primer pairs passed these initial screens and were further tested in two independent half-sib populations. Only one confirmed A. kolomikta primer pair, in cluster kC72369, was tested in an independent half-sib population. Ultimately, a primer pair from one cluster for each species, namely aC36306 and kC72369, displayed perfect sex linkage across all lines of their respective validation populations (Table 3).

The validated marker aC36306 amplifies a 106 bp band in $A$. arguta males with none to various alternate, presumably off-target fragments in females (Table 4; Supplemental information - File S1). When the aC36306 primers are multiplexed with a second universal forward primer nested between them, males are indicated by both an $81 \mathrm{bp}$ and a $106 \mathrm{bp}$ band, while females carry only a single $81 \mathrm{bp}$ band (Figure 1, top). The validated marker kC72369 amplifies a $161 \mathrm{bp}$ band in A. kolomikta males and a series of alternative loci in females (Table 4), with the brightest female band typically of a slightly higher molecular weight than the male band (Figure 1, bottom). Given the internal control included in the A. arguta marker and the consistent non-target amplification 
TABLE 1. Summary of de novo variant calling using GBS-SNP-CROP, along with descriptions of putative sex-linked variants.

\begin{tabular}{|c|c|c|c|c|c|c|c|c|c|c|c|c|}
\hline Species & Genotypes $^{a}$ & PE reads ${ }^{b}$ & $\begin{array}{c}\text { Total } \\
\text { clusters }^{\mathrm{c}}\end{array}$ & $\begin{array}{l}\text { Polymorphic } \\
\text { clusters }^{d}\end{array}$ & $\begin{array}{l}\text { Sex-linked } \\
\text { clusters }^{e}\end{array}$ & $\begin{array}{l}\text { Var. } \\
\text { type }^{f}\end{array}$ & $\begin{array}{c}\text { Var. } \\
\text { called }\end{array}$ & $\begin{array}{c}\text { Sex- } \\
\text { linked } \\
\text { var. }^{h}\end{array}$ & Depthi & $\begin{array}{c}\text { Hetero } \\
(\%)^{j}\end{array}$ & $\begin{array}{l}\text { Homo } \\
(\%)^{k}\end{array}$ & $\begin{array}{l}\text { NA } \\
(\%)^{\prime}\end{array}$ \\
\hline \multirow[t]{4}{*}{ A. arguta } & 59 & $286,335,086$ & 173,164 & 11,164 & 3 & SNPS & 30,468 & 2 & 59.99 & 27.82 & 60.17 & 11.99 \\
\hline & & & & & & indels & 1,754 & 1 & 64.58 & 24.42 & 65.15 & 10.41 \\
\hline & & & & & & Total & 32,222 & 3 & - & - & - & -- \\
\hline & & & & & & Mean & - & - & 60.24 & 27.63 & 60.44 & 11.93 \\
\hline \multirow[t]{4}{*}{ A. kolomikta } & 13 & $69,686,586$ & 115,680 & 6,719 & 4 & SNPS & 12,067 & 6 & 30.53 & 35.20 & 58.16 & 6.64 \\
\hline & & & & & & indels & 419 & 2 & 38.29 & 39.25 & 58.86 & 6.89 \\
\hline & & & & & & Total & 12,486 & 8 & - & - & - & -- \\
\hline & & & & & & Mean & - & - & 34.41 & 35.33 & 58.02 & 6.65 \\
\hline
\end{tabular}

a The number of accessions of known gender from each species.

b The total number of paired-end (PE) reads, considering all genotypes sampled.

c Total number of non-redundant consensus sequences (clusters) identified via clustering to represent the GBS fragment space used to build the reference sequence for alignment (i.e., the number of FASTA entries in the mock reference).

d The total number of polymorphic clusters.

e The number of clusters with putative sex-linked variants.

$f$ The type of variants called, either single nucleotide polymorphism (SNP) or insertion-deletion (indel).

$g$ The total number of variants called by the GBS-SNP-CROP pipeline.

h The number of putative sex-linked variants, by type.

I Average read depth for all variants across the entire population.

i Percentage of heterozygous genotype calls.

k Percentage of homozygous genotype calls.

I Percentage of missing cells (i.e., no genotype call for a given SNP*accession combination) in the final SNP genotype matrix.

TABLE 2. Details of the putative sex-linked variants (homozygous females, heterozygous males) identified via GBS association within the US collection of $60 \mathrm{~A}$. arguta and 13 A. kolomikta genotypes.

\begin{tabular}{|c|c|c|c|c|c|c|c|c|c|c|}
\hline Species & $\begin{array}{c}\text { Cluster } \\
I D^{a}\end{array}$ & $\begin{array}{l}\text { Length } \\
(\mathrm{bp})^{\mathrm{b}}\end{array}$ & $\begin{array}{l}\text { Var. } \\
\text { type }\end{array}$ & $\begin{array}{c}\text { Var. } \\
\text { position }\end{array}$ & $\begin{array}{l}\text { Female } \\
\text { homo }^{e}\end{array}$ & $\begin{array}{c}\text { Male } \\
\text { heterof }^{f}\end{array}$ & $\begin{array}{c}\text { Male } \\
\text { homog }\end{array}$ & $N A^{n}$ & $\begin{array}{c}\text { Female } \\
\text { genotype }\end{array}$ & $\begin{array}{c}\text { Male } \\
\text { genotype }\end{array}$ \\
\hline \multirow[t]{3}{*}{ A. arguta } & 70626 (1) & 128 & SNP & 115 & 40 & 15 & 2 & 3 & $\mathrm{C} / \mathrm{C}$ & $\mathrm{C} / \mathrm{T}$ \\
\hline & 36306 (1) & 157 & SNP* & 64 & 40 & 13 & 1 & 6 & $\mathrm{~T} / \mathrm{T}$ & $\mathrm{T} / \mathrm{C}$ \\
\hline & $123485(1)$ & 131 & indel $^{*}$ & 94 & 36 & 12 & 4 & 8 & $\mathrm{~A} / \mathrm{A}$ & A/-10GGTTTACCTT \\
\hline \multirow[t]{8}{*}{ A. kolomikta } & \multirow[t]{2}{*}{$693(1)$} & \multirow[t]{2}{*}{43} & SNP & 32 & 10 & 3 & 0 & 0 & $T / T$ & $\mathrm{~T} / \mathrm{G}$ \\
\hline & & & SNP & 6 & 10 & 3 & 0 & 0 & $\mathrm{G} / \mathrm{G}$ & $\mathrm{G} / \mathrm{A}$ \\
\hline & \multirow[t]{2}{*}{$1273(3)$} & \multirow[t]{2}{*}{203} & SNP & 17 & 10 & 3 & 0 & 0 & $T / T$ & $\mathrm{~T} / \mathrm{C}$ \\
\hline & & & indel & 189 & 10 & 3 & 0 & 0 & $T / T$ & T/-2TG \\
\hline & \multirow{2}{*}{$48400(2)$} & \multirow{2}{*}{221} & SNP* & 63 & 10 & 3 & 0 & 0 & $\mathrm{C} / \mathrm{C}$ & $\mathrm{C} / \mathrm{T}$ \\
\hline & & & SNP* & 153 & 10 & 3 & 0 & 0 & $\mathrm{C} / \mathrm{C}$ & $\mathrm{C} / \mathrm{T}$ \\
\hline & \multirow{2}{*}{$72369(2)$} & \multirow{2}{*}{270} & SNP & 57 & 10 & 3 & 0 & 0 & $\mathrm{~A} / \mathrm{A}$ & $\mathrm{A} / \mathrm{T}$ \\
\hline & & & indel $^{*}$ & 154 & 10 & 2 & 1 & 0 & $\mathrm{G} / \mathrm{G}$ & $\mathrm{G} /+3 \mathrm{ACC}$ \\
\hline
\end{tabular}

a The identification number of the GBS fragment (cluster/centroid) in the Mock Reference within which putative sex-linked variants were found.

Numbers in parentheses indicate the number of variants identified within each cluster.

b The total length of the GBS cluster, in base pairs.

c The type of variants called, either single nucleotide polymorphism (SNP) or insertion-deletion (indel). An asterisk ( ${ }^{*}$ ) indicates a variant around which primer pairs were designed.

d The variant position within the cluster, measured in bases from the start of the cluster sequence.

e The number of females scored as homozygotes. In this initial association analysis, there were a total of 42 and 8 female accessions for $A$. arguta and $A$. kolomikta, respectively.

$f$ The number of males scored as heterozygotes. In this initial association analysis, there were a total of 17 and 3 male accessions for $A$. arguta and $A$. kolomikta, respectively.

9 The number of males scored as homozygotes, potentially due to poor read depth. To be considered as potentially sex-linked, less than $5 \%$ of male lines were allowed to fall into this category.

${ }^{\mathrm{h}}$ The number of lines in the populations for which genotypes could not be inferred, due to insufficient GBS read depth. 
TABLE 3. Summary of PCR-inferred genders, relative to known genders, for lines in the initial populations as well as subsequent, independent validation populations for both species.

\begin{tabular}{|c|c|c|c|c|c|c|c|c|c|c|c|c|c|c|}
\hline \multirow{3}{*}{ Species } & \multirow{3}{*}{ Marker ID } & \multirow{2}{*}{\multicolumn{4}{|c|}{$\begin{array}{l}\text { Initial populations } \\
\text { US Collection of } \\
\text { A. arguta }(n=59)^{\text {a }}\end{array}$}} & \multicolumn{9}{|c|}{ Validation populations } \\
\hline & & & & & & \multicolumn{4}{|c|}{$\begin{array}{c}\text { OP progeny of } \\
\text { 'Ogden Point' }(n=17)\end{array}$} & \multicolumn{4}{|c|}{$\begin{array}{c}\text { OP progeny of } \\
\text { 'Hortgem Tahi' }(n=21)\end{array}$} & \multirow{2}{*}{$\begin{array}{l}\text { Fisher } \\
\text { Exact } p^{b}\end{array}$} \\
\hline & & $M$ & $\mathrm{~F}$ & $M_{R}$ & $F_{R}$ & $M$ & $\mathrm{~F}$ & $M_{R}$ & $F_{R}$ & $M$ & $\mathrm{~F}$ & $M_{R}$ & $F_{R}$ & \\
\hline \multirow[t]{2}{*}{ A. arguta } & aC36306 & 17 & 42 & 0 & 0 & 7 & 10 & 0 & 0 & 10 & 11 & 0 & 0 & $3.5 \times 10^{-11}$ \\
\hline & aC123485 & 14 & 42 & 3 & 0 & 5 & 8 & 2 & 0 & 9 & 10 & 2 & 0 & $1.6 \times 10^{-6}$ \\
\hline \multirow[t]{2}{*}{ Species } & \multicolumn{5}{|c|}{$\begin{array}{c}\text { US Collection of } \\
\text { A. kolomikta }(n=13)\end{array}$} & & & & & \multicolumn{4}{|c|}{$\begin{array}{l}\text { OP progeny of } \\
\text { 'Nahodka' }(n=21)\end{array}$} & Fisher \\
\hline & & $M$ & $\mathrm{~F}$ & $M_{R}$ & $F_{R}$ & & & & & $M$ & $\mathrm{~F}$ & $M_{R}$ & $F_{R}$ & \\
\hline A. kolomikta & kC72369 & 3 & 10 & 0 & 0 & & & & & 11 & 10 & 0 & 0 & $7.2 \times 10^{-10}$ \\
\hline
\end{tabular}

a Subscripted R's indicate recombination, or broken linkage, between the tested marker and the sex phenotype (e.g., $F_{R}$ is a phenotypic female that amplifies a male band).

${ }^{b}$ Fisher Exact Probability Test p-values for the validation populations.

TABLE 4. Primer and consensus GBS fragment (i.e., centroid) sequences for the validated, sex-linked markers in the two species A. arguta and A. kolomikta.

\begin{tabular}{|c|c|c|c|c|c|}
\hline Species & Primer IDa & Primer sequence ${ }^{b}$ & $\mathrm{Tm}^{\mathrm{c}}$ & $\begin{array}{l}\text { Band } \\
\text { size }\end{array}$ & $\begin{array}{c}\text { Marker } \\
\text { type }\end{array}$ \\
\hline \multirow{4}{*}{ A. arguta } & aC36306_male_for & TTTGGCAAAAACCACATgAC & \multirow{4}{*}{$52^{\circ} \mathrm{C}$} & \multirow{2}{*}{$106 \mathrm{bp}$} & Male \\
\hline & aC36306_univ_rev & AАAAGGGGGTTCGAACTTTG & & & Dominant \\
\hline & aC36306_univ_for & TGTTGGATGCCATTAAGTCG & & \multirow{2}{*}{$81 \mathrm{bp}$} & Internal \\
\hline & aC36306_univ_rev & AAAAGGGGGTTCGAACTTTG & & & Control \\
\hline
\end{tabular}

Cluster 36306 Paired Male and Female Sequences ${ }^{d}$

o 1 GTTAGCCAAAAAAATGGTAATATGTTACATTGGTACAATATCATTTTGGC

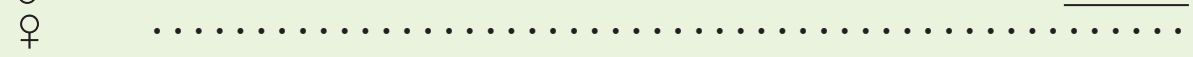

§ิ 51 AAAAACCACATCACCTTTCTGTTGGATGCCATTAAGTCGAAGCCGTCCAT

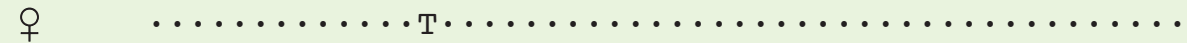

ઈิ 101 TGGTTGAAACAAGTATTCCCACСCTTGGATCAAAGTTCGAACССССTTTT

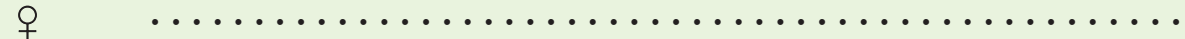

§ 151 AGGTCCG

우 $\quad \ldots \ldots$

\begin{tabular}{|c|c|c|c|c|c|}
\hline A. kolomikta & $\begin{array}{l}\text { kC72369_univ_for } \\
\text { kC72369 male rev }\end{array}$ & $\begin{array}{l}\text { TAGCCAAGGTAATGCCTCCA } \\
\text { САGGAАTATGTTCAAGAGTTGGT }\end{array}$ & $52^{\circ} \mathrm{C}$ & $161 \mathrm{bp}$ & $\begin{array}{c}\text { Male } \\
\text { Dominant }\end{array}$ \\
\hline
\end{tabular}

Cluster 72369 Paired Male and Female Sequences

ઈิ1 GCTTATGTTACCAAGCTAGCCAAGGTAATGCCTCCACCAACATTTTCTTT

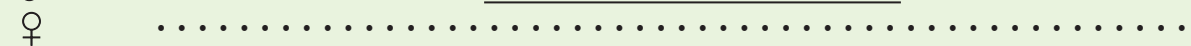

§ 51 АTATTTACACGTCTTAGTTTGATTATTTTTGTACAGAGACTTGACTCCGA

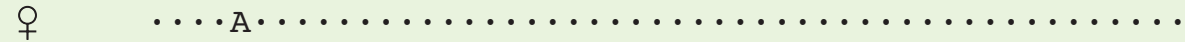

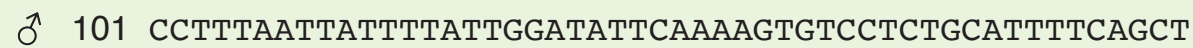

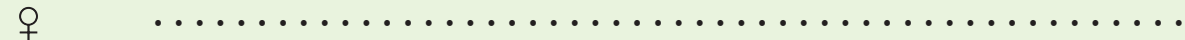

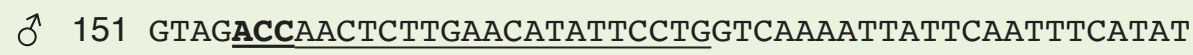

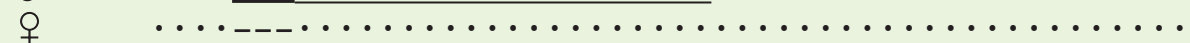

ऽ 201 GCAATTCAATAGAAAATTACAAAGTTTTTAACTCAATGAGAGAGATTGTG

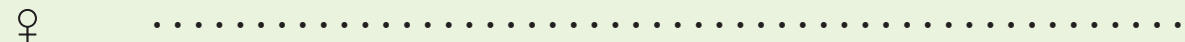

§ 251 TAGATTAСTTTTTATTCCTGTCT

o $\quad \ldots \ldots \ldots \ldots \ldots \ldots \ldots \ldots \ldots \ldots$

a Within the Primer IDs, "male" means the primer is male-specific, "univ" means the primer amplifies both male and female alleles, "for" = forward primer, and "rev" = reverse primer.

${ }^{b}$ Nucleotides in bold are male-specific; lower-case nucleotides are degenerate, matching neither male nor female alleles.

c Optimal annealing temperatures $(\mathrm{Tm})$ were determined via temperature gradient trials.

d Underlined sequences indicate the locations of the primers in the centroids; a dot $(\cdot)$ in the female-specific sequences indicates consensus with the male-specific base; a dash (-) in the female-specific sequences indicates a deletion, relative to the male-specific base. 
in A. kolomikta females, both markers are effectively co-dominant, helping to reduce inaccurate scoring due to PCR failures. Neither marker was found to exhibit sex-linkage in the other species (data not shown).

When used as BLAST query sequences against the A. chinensis var. chinensis draft genome (Huang et al., 2013), the cluster sequences of both markers align to regions in the "Unknown" pseudomolecule, with the amplified regions of clusters aC36306 and kC72369 aligning at 97\% and 92\% identity, respectively. Neither marker aligns to known coding regions or possesses significant similarity to either repetitive or non-coding sequences in the NCBI database.

\section{Discussion}

As the quality and affordability of genome sequencing technologies continue to improve, a practical challenge facing marker-assisted breeders is how to use such data to efficiently identify trait-associated polymorphisms amenable to conversion into cost-effective molecular markers for screening large populations. This bioinformatic challenge of mining large genomic datasets for potential low-cost markers is amplified for breeders of minor and orphan crops due to there often being: 1) Little to no genomic resources available (e.g., high-quality reference genomes) to facilitate marker discovery; and 2) Considerable resource constraints compared to breeding programs for major crops. This study demonstrates the practical use of the GBS-SNP-CROP pipeline (Melo et al., 2016) to discover trait-linked polymorphisms (both SNPs and indels) in the absence of a reference genome. Candidate polymorphisms were converted into low-cost, gel-based PCR markers and validated in independent populations, thereby providing a concrete example of the successful use of de novo GBS analysis for marker development in minor crops, both diploid (A. kolomikta) and tetraploid (A. arguta).

Given their consistent homozygosity in females and heterozygosity in males, the markers developed and validated in this study support the ploidy-independent, Y-active sex inheritance system observed in A. chinensis var. chinensis and A. chinensis var. deliciosa (Gill et al., 1998; Fraser et al., 2009; Testolin et al., 1995, 1999). While breeding programs for these more globally important kiwifruit species have benefitted from the development of sex-linked markers for many years (Harvey et al., 1997; Shirkot et al., 2002; Zhang et al., 2015), analogous markers for A. arguta and A. kolomikta have been lacking. The perfect sex-linkages of the two reported markers across the populations in this study suggest they may be used to confidently score progeny in cold-hardy kiwifruit breeding programs, resulting in a significant increase in resource efficiency. Specifically, given the slight male bias observed among populations of $A$. arguta progeny (Supplemental information - Table S2), the use of marker a36306 at the seedling stage should allow programs to effectively double the sizes of their breeding populations without driving up overall costs.

Due to the 2010 ban on the importation of Actinidia germplasm into the US to prevent the introduction of the pathogen Pseudomonas syringae pv. actinidiae (USDA, 2010), this study focused predominantly on lines within the US collection. While the relevance of these markers to other germplasm collections of A. arguta and A. kolomikta should be confirmed before they are adopted by non-US breeding programs, the validation of marker a36306 in the OP population from cv. Hortgem Tahi is encouraging. Not only is 'Hortgem Tahi' not in the US collection, its population of half-sib progeny likely represent even more non-US genetic diversity as they were derived from seeds of store-bought berries produced in and imported from New Zealand. If breeding programs discover that the markers reported here do not amplify in certain populations, the other sex-associated sequences discovered by this study (Supplemental information - File S1) may prove useful in fast-tracking the development of alternative markers, including CAPS markers.

Finally, it is worth noting that the clarity of gel-based markers depends not only on the inherent properties of the sequences involved, but also on the DNA extraction method and the PCR cycling conditions used. The method of DNA isolation used here sufficed for the development and validation of the reported markers, but PCR efficiency was seen to vary across batches. While amplification inhibition, when observed, was typically remedied through either a reduction of template (i.e., genomic DNA) concentration in the PCR reaction and/or an increase in cycle number, medium-to-high-throughout molecular screens require uniformity. To that end, potential users of these markers are

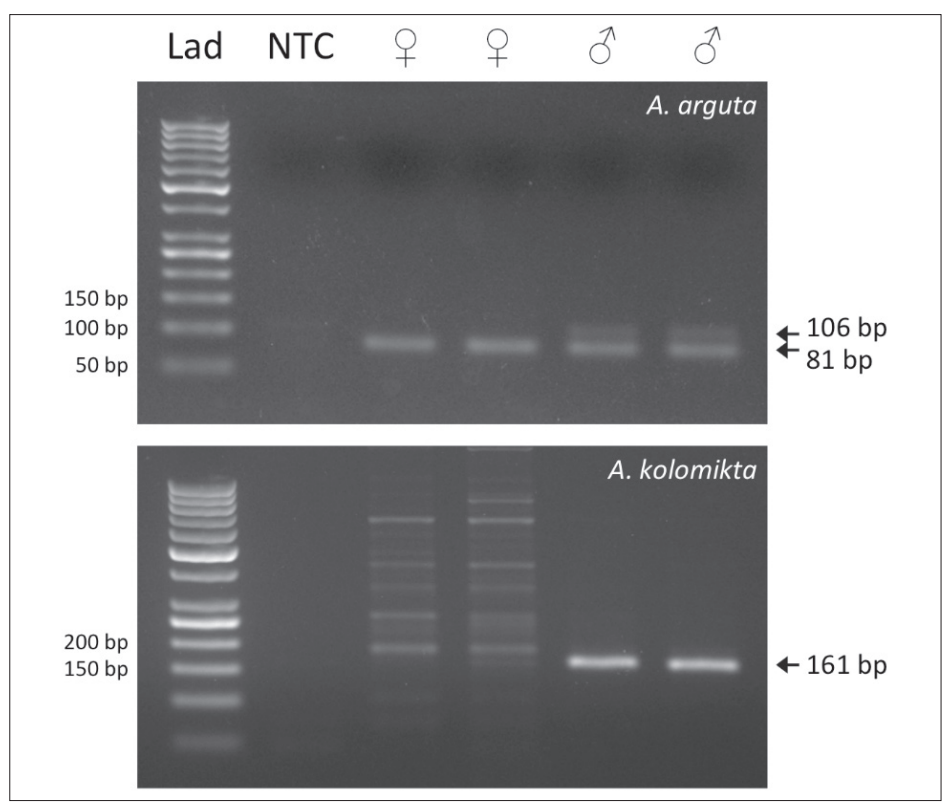

Figure 1. Agarose (2\%) gel electrophoresis of the PCR products of $A$. arguta marker aC36306 with an 81 bp internal control (top) and A. kolomikta marker kC72369 (bottom). The relative faintness of the $81 \mathrm{bp}$ band in the upper gel is inherent to the Y-active sex inheritance system of Actinidia spp. For tetraploid $A$. arguta, the male (Y) allele is expected to be present at a quarter of the dosage (i.e., onefourth the brightness) of control (i.e., universal) alleles. $\mathrm{Lad}=50 \mathrm{bp}$ Gene Ruler, NTC = non-template control, $q=$ female, $\delta^{\lambda}=$ male. 
encouraged to collect young leaf tissue and invest effort in optimizing the extraction process under their specific laboratory conditions.

\section{Acknowledgments}

We are indebted to W. Hastings for field support; R. Bartaula for laboratory technical support; R. Guthrie for leaf tissue of the Ogden Point and Nahodka OP populations; and D. Jackson and H. Laubach of Kiwi Berry Organics Co. (Danville, PA) for leaf tissue of the 'Hortgem Tahi' OP population. Partial funding was provided by the New Hampshire Agricultural Experiment Station. This is Scientific Contribution Number 2716. This work is supported by the USDA National Institute of Food and Agriculture, Multi-State Hatch Project 233561.

\section{Conflicts of interest}

The authors declare that there are no conflicts of interest.

\section{References}

Ainsworth, C.C. (1999). Sex Determination in Plants, $1^{\text {st }}$ edn. (Oxford: BIOS Scientific Publishers Ltd.)

Bolger, A.M., Lohse, M., and Usadel, B. (2014). Trimmomatic: A flexible trimmer for Illumina sequence data. Bioinformatics 30, 2114-2120. https://doi.org/10.1093/bioinformatics/btu170.

Crowhurst, R.N., Gleave, A.P., MacRae, E.A., Ampomah-Dwamena, C., Atkinson, R.G., Beuning, L.L., et al. (2008). Analysis of expressed sequence tags from Actinidia: applications of a cross species EST database for gene discovery in the areas of flavor, health, color and ripening. BMC Genomics 9, 351. https://doi.org/10.1186/14712164-9-351.

Deputy, J.C., Ming, R., Ma, H., Liu, Z., Fitch, M., Wang, M., Manshardt, R., and Stiles, J.I. (2002). Molecular markers for sex determination in papaya (Carica papaya L.). Theor. Appl. Genet. 106, 107-111. https://doi.org/10.1007/s00122-002-0995-0.

Ferguson, A.R. (2013). Kiwifruit. The wild and the cultivated plants. Adv. Food. Nutr. Res. 68, 15-32. https://doi.org/10.1016/B978-012-394294-4.00002-X.

Ferguson, A.R., and Huang, H.W. (2007). Genetic resources of kiwifruit: domestication and breeding. Hortic. Rev. 1-121. https:// doi.org/10.1002/9780470168011.ch1.

Ferguson, A.R., and Seal, A.G. (2008). Kiwifruit. In Temperate Fruit Crop Breeding, J.F. Hancock, ed. (New York: Springer), p. 235-263. https://doi.org/10.1007/978-1-4020-6907-9_8.

Food and Agriculture Organization of the United Nations (FAO) (2016). FAOSTAT Statistics Database. http://faostat3.fao.org (accessed November 14, 2017)

Fraser, L.G., Tsang, G.K., Datson, P.M., De Silva, H.N., Harvey, C.F., Gill, G.P., Crowhurst, R.N., and McNeilage, M.A. (2009). A gene-rich linkage map in the dioecious species Actinidia chinensis (kiwifruit) reveals putative X/Y sex-determining chromosomes. BMC Genomics 10, 102. https://doi.org/10.1186/1471-2164-10-102.

Friis, E.M., Chaloner, W.G., and Crane, P.K. (1987). The origins of Angiosperms and their biological consequences, $1^{\text {st }}$ edn. (New York: Cambridge University Press).

Gill, G.P., Harvey, C.F., Gardner, R.C., and Fraser, L.G. (1998). Development of sex-linked PCR markers for gender identification in Actinidia. Theor. Appl. Genet. 97, 439-445. https://doi.org/10.1007/ s001220050914.

Goodale, G.L. (1981). Useful Plants of the Future. Some of the Possibilities of Economic Botany, $1^{\text {st }}$ edn. (Salem, MA: Salem Press Publishing and Printing Co.).
Harvey, C.F., Gill, G.P., Fraser, L.G., and McNeilage, M.A. (1997). Sex determination in Actinidia. 1. Sex-linked markers and progeny sex ratio in diploid A. chinensis. Sexual Plant Reprod. 10, 149-154. https://doi.org/10.1007/s004970050082.

Huang, S., Ding, J., Deng, D., Tang, W., Sun, H., et al. (2013). Draft genome of the kiwifruit Actinidia chinensis. Nat. Commun. 4, 2640. https://doi.org/10.1038/ncomms3640.

Li, H., and Durbin, R. (2009). Fast and accurate short read alignment with Burrows-Wheeler Transform. Bioinformatics 25, 1754-1760. https://doi.org/10.1093/bioinformatics/btp324.

Li, H., Handsaker, B., Wysoker, A., Fennell, T., Ruan, J., Homer, J., Marth, G., Abecasis, G., and Durbin, R. (2009). The Sequence Alignment/ Map format and SAMtools. Bioinformatics 25, 2078-2079. https:// doi.org/10.1093/bioinformatics/btp352.

Mandolino, G., Carboni, A., Forapani, S., Faeti, V., and Ranalli, P. (1999). Identification of DNA markers linked to the male sex in dioecious hemp (Cannabis sativa L.). Theor. Appl. Genet. 98, 86-92. https://doi.org/10.1007/s001220051043.

Melo, A.T., Guthrie, R.S., and Hale, I. (2017). GBS-based deconvolution of the surviving North American collection of cold-hardy kiwifruit (Actinidia spp.) germplasm. PloS One 12(1). https://doi. org/10.1371/journal.pone.0170580.

Melo, A.T.O., Bartaula, R., and Hale, I. (2016). GBS-SNP-CROP: a reference-optional pipeline for SNP discovery and plant germplasm characterization using variable length, paired-end genotypingby-sequencing data. BMC Bioinformatics 17, 29. https://doi. org/10.1186/s12859-016-0879-y.

Ming, R., Bendahmane, A., and Renner, S.S. (2011). Sex chromosomes in land plants. Annu. Rev. Plant. Biol. 62, 485-514. https://doi. org/10.1146/annurev-arplant-042110-103914.

Nishiyama, I., Yamashita, Y., Yamanaka, M., Shimohashi, A., Fukuda, T., and Oota, T. (2004). Varietal difference in vitamin C content in the fruit of kiwifruit and other Actinidia species. J. Agric. Food. Chem. 52, 5472-5475. https://doi.org/10.1021/jf049398z.

Poland, J., Brown, P.J., Sorrells, M.E., and Jannink, J.L. (2012). Development of high-density genetic maps for barley and wheat using a novel two-enzyme genotyping-by-sequencing approach. PLoS One 7(2). https://doi.org/10.1371/journal.pone.0032253.

Reamon-Buettner, S.M., and Jung, C. (2000). AFLP-derived STS markers for the identification of sex in Asparagus officinalis L. Theor. Appl. Genet. 100, 432-438. https://doi.org/10.1007/ s001220050056.

Renner, S.S., and Ricklefs, R.E. (1995). Dioecy and its correlates in the flowering plants. Am. J. Bot. 82, 596-606. https://doi. org/10.1002/j.1537-2197.1995.tb11504.x.

Rozen, S., and Skaletsky, H.J. (2000). Primer3 on the WWW for general users and for biologist programmers. In Bioinformatics Methods and Protocols: Methods in Molecular Biology, S. Krawetz, and S. Misener, eds. (Totowa, NJ: Humana Press), p. 365-386.

Seal, A.G., and McNeilage, M.A. (1988). Sex and kiwifruit breeding. Australian Temperate Fruits Review Conference 240, 35-38.

Shirkota, P., Sharmaa, D.R., and Mohapatrab, T. (2002). Molecular identification of sex in Actinidia deliciosa var. deliciosa by RAPD markers. Sci. Hortic. 94, 33-39. https://doi.org/10.1016/S03044238(01)00357-0.

Testolin, R., Cipriani, G., and Costa, G. (1995). Sex segregation ratio and gender expression in the genus Actinidia. Sexual Plant Reprod. 8 129-132. https://doi.org/10.1007/BF00242255.

Testolin, R., Cipriani, G., and Messina, R. (1999). Sex control in Actinidia is monofactorial and remains so in polyploids. In Sex Determination in Plants, C.C. Ainsworth, ed. (Oxford: BIOS Scientific Publishers Ltd.), p. 175-184. 
USDA APHIS PPQ (2010). Federal Order for Pseudomonas syringae pv. actinidiae, bacterial canker of kiwifruit. In USDA APHIS Federal Import Quarantine Order. http://nationalplantboard.org/wpcontent/uploads/docs/spro/spro_bck_2010_11_10.pdf.

Zhang, Q., Liu, C., Liu, Y., VanBuren, R., Yao, X., Zhong, C., and Huang, H. (2015). High-density interspecific genetic maps of kiwifruit and the identification of sex-specific markers. DNA Res. 22(5), 367-375. https://doi.org/10.1093/dnares/dsv019.

Received: May 16, 2017

Accepted: Nov. 28, 2017
Addresses of authors:

Iago Hale*, Arthur T.O. Melo and Haley Gustafson

University of New Hampshire, College of Life Science and

Agriculture, Department of Agriculture, Nutrition, and Food Systems, Durham, NH, USA

* Corresponding author; E-mail: iago.hale@unh.edu; Tel.: 1-603-862-4653

SUPPlEMENTAL INFORMATION - TABle S1. List of the 60 non-redundant accessions of Actinidia arguta and the 13 nonredundant accessions of $A$. kolomikta used for the initial screening of sex-linked variants.

\begin{tabular}{|c|c|c|c|c|c|c|c|c|c|c|}
\hline No. & Genotype name & $\mathrm{PI} \#$ & САCТ \# & DACT\# & $\begin{array}{l}\text { Gender } \\
\text { reported }\end{array}$ & $\begin{array}{l}\text { Gender } \\
\text { observed }\end{array}$ & $\begin{array}{c}\text { GBS } \\
\text { barcode }\end{array}$ & $\begin{array}{l}\mathrm{PE} \\
\text { reads }\end{array}$ & $\begin{array}{l}\text { Variants } \\
\text { called }\end{array}$ & $\begin{array}{c}\text { SRA } \\
\text { number }\end{array}$ \\
\hline \multicolumn{11}{|c|}{ Actinidia arguta } \\
\hline 1 & $\# 211 \mathrm{~A}$ & 637808 & 169 & 272 & M & M & CATAT & 6401008 & 30173 & SRR3234153 \\
\hline 2 & \#211B & 637809 & 170 & 271 & - & $\mathrm{F}$ & CACCA & 5959902 & 30175 & SRR3234114 \\
\hline 3 & \#74 Female & 637804 & 164 & 326 & $\mathrm{~F}$ & $\mathrm{~F}$ & АTCTGT & 4478434 & 29507 & SRR3234194 \\
\hline 4 & $119-40$ & 617156 & 108 & 202 & $\mathrm{~F}$ & $\mathrm{~F}$ & TGCCGCAT & 3857758 & 29020 & SRR3234174 \\
\hline 5 & $125-40$ & 617157 & 109 & 204 & $\mathrm{~F}$ & - & GGCTTA & 3554890 & 29366 & SRR3234179 \\
\hline 6 & $127-40$ & 617142 & 92 & 206 & M & $\mathrm{F}$ & TAGCGGAT & 1087034 & 17411 & SRR3234154 \\
\hline 7 & $127-40$ & 617163 & 120 & 205 & M & M & CCTTGCCATT & 4746724 & 29922 & SRR3234196 \\
\hline 8 & $74-32$ & 617113 & 54 & 127 & M & M & GGTGCACATT & 5488740 & 29999 & SRR3234121 \\
\hline 9 & $74-46^{*}$ & - & - & - & M & M & GGAACGA & 4387350 & 29503 & SRR3234155 \\
\hline 10 & $74-49$ & 617103 & 43 & 208 & $\mathrm{~F}$ & $\mathrm{~F}$ & CTCGCGG & 4739038 & 30092 & SRR3234197 \\
\hline 11 & $74-52$ & - & - & - & M & M & AACGTGCCT & 3936772 & 29447 & SRR3234193 \\
\hline 12 & $74-55$ & 617114 & 56 & 132 & $\mathrm{~F}$ & $\mathrm{~F}$ & TATCA & 3337274 & 29293 & SRR3234177 \\
\hline 13 & Ananasnaya & 617104 & 44 & 220 & $\mathrm{~F}$ & $\mathrm{~F}$ & CGTGTCA & 3368520 & 28981 & SRR3234178 \\
\hline 14 & Andrey & - & - & - & M & M & TATTCGCAT & 4494836 & 29621 & SRR3234159 \\
\hline 15 & Chang Bai Mountain 1 & 617110 & 50.001 & 212 & $\mathrm{~F}$ & $\mathrm{~F}$ & GCTCCGA & 397712 & 8016 & SRR3234135 \\
\hline 16 & Chang Bai Mountain 2 & 617110 & 50.002 & 212 & $\mathrm{~F}$ & $\mathrm{~F}$ & CTCGCGG & 3433118 & 29174 & SRR3234117 \\
\hline 17 & Chang Bai Mountain 3 & 617110 & 50.003 & 212 & $\mathrm{~F}$ & $\mathrm{~F}$ & CAGATA & 11533362 & 30487 & SRR3234099 \\
\hline 18 & Chang Bai Mountain 4 & 617110 & 50.004 & 212 & M & M & CCTTGCCATT & 8735374 & 30356 & SRR3234104 \\
\hline 19 & Chang Bai Mountain 5 & 617110 & 50.005 & 212 & $\mathrm{~F}$ & $\mathrm{~F}$ & ACTGCGAT & 1855156 & 25457 & SRR3234111 \\
\hline 20 & Chico & 667888 & 114 & 223 & $\mathrm{~F}$ & $\mathrm{~F}$ & ACAACT & 4743214 & 29847 & SRR3234161 \\
\hline 21 & Cornell female & - & - & - & $F$ & $\mathrm{~F}$ & GCCTACCT & 3747072 & 29727 & SRR3234113 \\
\hline 22 & DACT 123 & $617152 / 667991$ & 104 & 123 & $\mathrm{~F}$ & $\mathrm{~F}$ & CAAGT & 5751718 & 30157 & SRR3234158 \\
\hline 23 & DACT 213 & 637813 & 175 & 213 & $\mathrm{~F}$ & M & ACAGT & 4723552 & 30036 & SRR3234199 \\
\hline 24 & DACT 214 & 637812 & 174 & 214 & $\mathrm{~F}$ & $\mathrm{~F}$ & AACTGG & 5322144 & 30231 & SRR3234139 \\
\hline 25 & DACT 216 & 637811 & 172 & 216 & $\mathrm{~F}$ & $\mathrm{~F}$ & GTCGCCT & 3793736 & 29231 & SRR3234163 \\
\hline 26 & DACT 218 & 637810 & 171 & 218 & $\mathrm{~F}$ & $\mathrm{~F}$ & TTGCTG & 5227524 & 29912 & SRR3234183 \\
\hline 27 & DACT 260 & 617168 & 139 & 260 & $\mathrm{~F}$ & $\mathrm{~F}$ & TATGT & 8100576 & 30118 & SRR3234165 \\
\hline 28 & Dumbarton Oaks* & 617135 & 84 & 225 & $\mathrm{~F}$ & $\mathrm{~F}$ & GAATGCAATA & 6049356 & 29825 & SRR3234143 \\
\hline 29 & Early Cordifolia & - & - & - & $\mathrm{F}$ & $\mathrm{F}$ & ATAGAT & 4634780 & 29835 & SRR3234167 \\
\hline 30 & Flowercloud male* & - & - & - & M & M & ATTAT & 1606094 & 23072 & SRR3234109 \\
\hline 31 & Frenchman's Bay & 617162 & 116 & 226 & $\mathrm{~F}$ & $\mathrm{~F}$ & GGTGT & 6368934 & 29962 & SRR3234107 \\
\hline 32 & Geneva 1 & 617101 & 30 & $040 / 227$ & $\mathrm{~F}$ & $\mathrm{~F}$ & ATATAA & 1651662 & 22389 & SRR3234186 \\
\hline 33 & Geneva 3 & 617133 & 80 & 229 & $\mathrm{~F}$ & $\mathrm{~F}$ & TCTTGG & 6276020 & 30075 & SRR3234144 \\
\hline 34 & Hardy Red & 617107 & 47 & 230 & $\mathrm{~F}$ & $\mathrm{~F}$ & AACGTGCCT & 4646988 & 29359 & SRR3234124 \\
\hline
\end{tabular}


SUPPLEMENTAL INFORMATION - TABLE S1. (Continued).

\begin{tabular}{|c|c|c|c|c|c|c|c|c|c|c|}
\hline No. & Genotype name & $\mathrm{PI} \#$ & САCT\# & DACT \# & $\begin{array}{l}\text { Gender } \\
\text { reported }\end{array}$ & $\begin{array}{c}\text { Gender } \\
\text { observed }\end{array}$ & $\begin{array}{c}\text { GBS } \\
\text { barcode }\end{array}$ & $\begin{array}{l}\mathrm{PE} \\
\text { reads }\end{array}$ & $\begin{array}{l}\text { Variants } \\
\text { called }\end{array}$ & $\begin{array}{c}\text { SRA } \\
\text { number }\end{array}$ \\
\hline 35 & HVSC-115* & 641101 & 186 & 231 & $F$ & $\mathrm{~F}$ & ATGAGCAA & 2840748 & 27899 & SRR3234118 \\
\hline 36 & HVSC-117 & 641102 & 188 & 232 & - & M & CAACCACACA & 3209702 & 28933 & SRR3234134 \\
\hline 37 & Issai & 617106 & 46 & 233 & $\mathrm{~F}$ & $\mathrm{~F}$ & GAAGCA & 4525900 & 27956 & SRR3234123 \\
\hline 38 & Issai small fruit variant & $617116 / 667909$ & 58 & 134 & $\mathrm{~F}$ & $\mathrm{~F}$ & ATATCGCCA & 3151572 & 27660 & SRR3234120 \\
\hline 39 & Jumbo & 617108 & 48 & 234 & $\mathrm{~F}$ & $\mathrm{~F}$ & ATGAGCAA & 5544624 & 30045 & SRR3234146 \\
\hline 40 & Male Hardy Kiwi & - & - & - & M & M & CAAGT & 4940840 & 29546 & SRR3234125 \\
\hline 41 & Michigan State & 617136 & 86 & 239 & $\mathrm{~F}$ & $F$ & GGTGT & 5521514 & 30052 & SRR3234149 \\
\hline 42 & New Zealand & 617125 & 70 & 334 & $\mathrm{~F}$ & $\mathrm{~F}$ & TGGCAACAGA & 965764 & 20792 & SRR3234191 \\
\hline 43 & Ogden Point* & 617140 & 90 & 240 & $\mathrm{~F}$ & $\mathrm{~F}$ & AACGCACATT & 2749792 & 28370 & SRR3234136 \\
\hline 44 & Opitz & 637803 & 163 & 241 & M & M & GGTATA & 5665492 & 30211 & SRR3234105 \\
\hline 45 & Opitz Male* & 637802 & 162 & 242 & M & M & TCTTGG & 6587636 & 30120 & SRR3234106 \\
\hline 46 & ORUS 1-3 & 667903 & 254 & 244 & - & M & GGAAGACAT & 3857518 & 29431 & SRR3234102 \\
\hline 47 & ORUS 1-4 & 667890 & 239 & 322 & - & $F$ & TGCTT & 4645838 & 29675 & SRR3234116 \\
\hline 48 & ORUS 1-5 & 667899 & 250 & 245 & - & $\mathrm{F}$ & TCACTG & 4455038 & 28879 & SRR3234127 \\
\hline 49 & ORUS 1-6 & 667889 & 238 & 246 & - & $\mathrm{F}$ & CATAT & 11782074 & 30300 & SRR3234130 \\
\hline 50 & ORUS 1-8* & 667904 & 255 & 247 & - & M & ACAGT & 2096570 & 25534 & SRR3234128 \\
\hline 51 & ORUS 2-1 & 667905 & 256 & 248 & M & M & CTTGA & 8998044 & 30153 & SRR3234151 \\
\hline 52 & ORUS 2-16 [MR] & 667891 & 241 & 249 & $\mathrm{~F}$ & $\mathrm{~F}$ & TAGCGGAT & 12638534 & 30824 & SRR3234100 \\
\hline 53 & ORUS 2-17* & 667901 & 252 & 250 & - & M & GGATA & 2219262 & 25952 & SRR3234108 \\
\hline 54 & ORUS 2-3 & 667900 & 251 & 333 & - & $\mathrm{F}$ & СTCTA & 4351174 & 29586 & SRR3234192 \\
\hline 55 & ORUS 2-7* & 667895 & 245 & 252 & - & $\mathrm{F}$ & CATCTGCCG & 2135604 & 25584 & SRR3234132 \\
\hline 56 & ORUS 3-3 & 667892 & 242 & 254 & - & $\mathrm{F}$ & GGACAG & 8156828 & 30153 & SRR3234133 \\
\hline 57 & Red Princess & 617118 & 60 & 274 & $\mathrm{~F}$ & $\mathrm{~F}$ & AАТАACCAA & 3386808 & 29338 & SRR3234122 \\
\hline 58 & Smith 2 Female & 617130 & 75 & 255 & - & - & TGGCACAGA & 4624310 & 29932 & SRR3234176 \\
\hline 59 & Tatyana & - & - & - & $\mathrm{F}$ & $\mathrm{F}$ & ATATAA & 1469580 & 23415 & SRR3234112 \\
\hline 60 & West $^{*}$ & 617121 & 66 & 257 & $\mathrm{~F}$ & $\mathrm{~F}$ & GCGTCCT & 6337356 & 29702 & SRR3234156 \\
\hline \multicolumn{11}{|c|}{ Actinidia kolomikta } \\
\hline 1 & $\mathrm{~A}-10^{*}$ & 617145 & 95 & 275 & $\mathrm{~F}$ & $\mathrm{~F}$ & GTGACACAT & 7989260 & 12078 & SRR3234080 \\
\hline 2 & Ananasnaya & - & - & - & $\mathrm{F}$ & $\mathrm{F}$ & AAGACGCT & 4765536 & 11872 & SRR3234096 \\
\hline 3 & Dr. Szymanowski & 617166 & 137 & 280 & $\mathrm{~F}$ & $\mathrm{~F}$ & GGCTTA & 5907658 & 11703 & SRR3234072 \\
\hline 4 & Frost & - & - & - & $\mathrm{F}$ & - & CGCACCAATT & 5687430 & 12086 & SRR3234093 \\
\hline 5 & Klara Zetkin* & - & - & - & $\mathrm{F}$ & $\mathrm{F}$ & ATATCGCCA & 3464698 & 11699 & SRR3234091 \\
\hline 6 & Krupnopladnaya & 617122 & 67 & 282 & $\mathrm{~F}$ & M & ATCTGT & 3596006 & 10868 & SRR3234071 \\
\hline 7 & Hero & - & - & - & $\mathrm{F}$ & - & TCACGGAAG & 6626818 & 11587 & SRR3234085 \\
\hline 8 & $\begin{array}{l}\text { Leningradskaya } \\
\text { ozdnaya* }^{*}\end{array}$ & 617150 & 101 & 285 & M & M & CGTTCA & 5341386 & 12025 & SRR3234097 \\
\hline 9 & Matovnaya [MR] & 617147 & 97 & 286 & $\mathrm{~F}$ & $\mathrm{~F}$ & CAGTGCCATT & 9769392 & 12120 & SRR3234083 \\
\hline 10 & Nahodka & 617120 & 65 & 287 & $\mathrm{~F}$ & $\mathrm{~F}$ & TTCGTT & 2343654 & 10159 & SRR3234075 \\
\hline 11 & Oluyckos* & 641095 & 127 & 289 & $\mathrm{~F}$ & $\mathrm{~F}$ & CGTGGACAGT & 4791980 & 11982 & SRR3234084 \\
\hline 12 & Raintree* $^{*}$ & 617126 & 71 & 292 & M & M & TCACTG & 5763436 & 11940 & SRR3234094 \\
\hline 13 & Urozhainaya & 641096 & 129 & 295 & - & $\mathrm{F}$ & GAGCGACAT & 3639332 & 11426 & SRR3234073 \\
\hline
\end{tabular}

For each accession, the following information is provided:

1 Accession name $\left(^{*}=\right.$ genotypes used for initial PCR-based marker testing; [MR] $=$ the female used to build each species-specific Mock Reference, due to its high number of PE reads).

2 USDA Plant Introduction (PI) number(s), if assigned.

3 USDA Corvallis Actinidia (CACT) accession number, if assigned.

4 USDA Davis Actinidia (DACT) accession number(s), if assigned.

5 Reported gender.

6 Gender observed at UNH (Note: In cases of conflict between the reported gender and the gender observed at UNH, the observed gender of the plant from which DNA was isolated was used).

7 GBS barcode assignment.

8 Number of high-quality paired-end (PE) reads used to call SNPS.

9 Number of variants (SNPs and indels) called.

10 Assigned NCBI Sequence Read Archive (SRA) number. 
SUPPLEMENTAL INFORMATION - TABLE S2. Genotype-inferred genders of full-sib progeny from 13 A. arguta crosses, based on the sex-linked marker aC36306.

\begin{tabular}{lccc}
\hline Population (mother × father) & Males & Females & \% Males \\
\hline Dumbarton Oaks × Russia \#114 & 19 & 11 & 63.3 \\
UCD 3-1 × CACT 160 \#111 & 47 & 17 & 73.4 \\
Dumbarton Oaks × Chang Bai Mountain 4 & 5 & 5 & 50.0 \\
Tatyana × 74-32 & 23 & 16 & 59.0 \\
Ogden Point × CACT 105 \#107 & 74 & 54 & 57.8 \\
Ogden Point × C-5 (HRC) & 88 & 74 & 54.3 \\
UCD 3-1 × C-5 (HRC) & 19 & 22 & 46.3 \\
Dumbarton Oaks × C-5 (HRC) & 56 & 65 & 46.3 \\
Chang Bai Mountain 5 × 74-32 & 61 & 53 & 53.5 \\
Dumbarton Oaks × CACT 105 \#107 & 68 & 60 & 53.1 \\
DACT 218 × CACT 105 \#107 & 20 & 15 & 57.1 \\
Ogden Point × Chang Bai Mountain 4 & 68 & 75 & 47.6 \\
Ogden Point × CACT 160 \#111 & 65 & 51 & 56.0 \\
\hline Totals & 613 & 518 & 55.2 \\
Mean & & & \\
\hline
\end{tabular}

SUPPlEMENTAL INFORMATion - File S1. Paired FASTA entries describing the nucleotide sequences of homozygous female and heterozygous male genotypes for putative sex-linked variants, based on the initial GBS association analyses.

FASTA header structure:

$>$ GBS cluster ID within the Mock Reference | Cluster length (bp) | Polymorphism type | Mock Reference allele | Polymorphism position within the cluster (bp) | Female/Male genotype.

Actinidia arguta

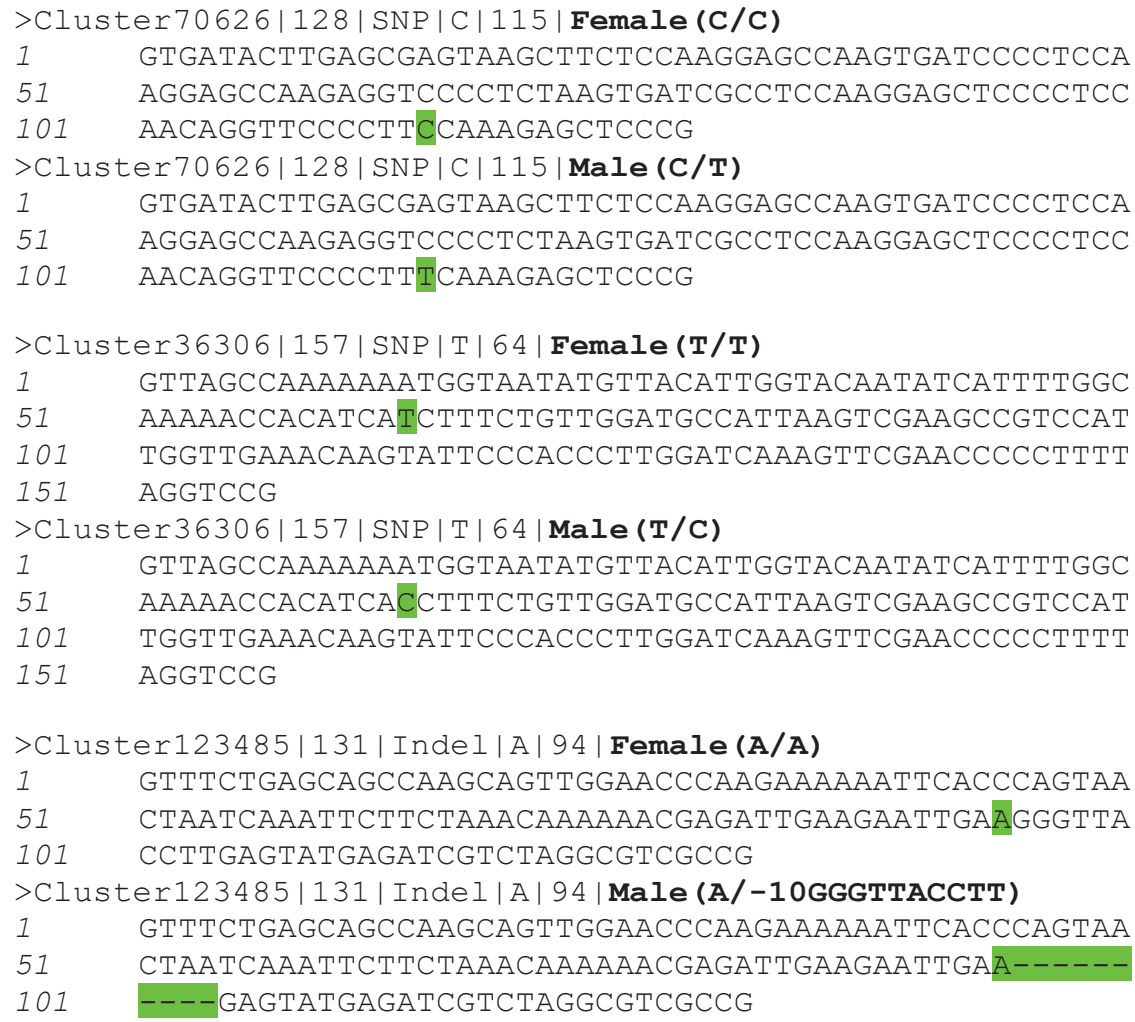


SUPPLEMENTAL INFORMATION - File S1. (Continued).

Actinidia kolomikta

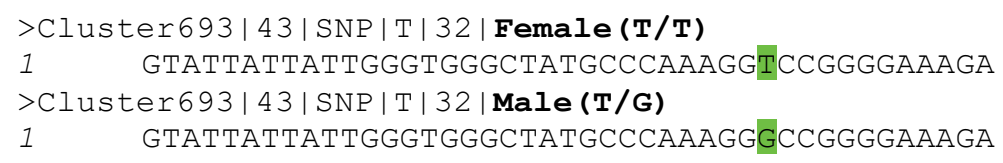

>Cluster1273|203|SNP|G|6|Female (G/G)

>Cluster1273|203|SNP|T|17| Female (T/T)

>Cluster1273|203|Indel|T|189|Female(T/T)

1 GGTACGGCTGTTATGATGTCCAGAGGGTTGGTTTTGCAATAATTGCAGAC

51 TTTCTCCTTAGAGCAGCTCAGATTGCGAGTGCAGTACAAAGGGAAAATGG

101 CACGTTGTAGAAACAATTCCTGAAGCCTGAAGGGTCTAAAAACAATATAA

151 GTATTGATGAGCTCCGAAACAAGTTTGAGACGTTTGCATTGTGCCCCGGG

201 GAA

$>$ Cluster1273|203|SNP|G|6|Male (G/A)

>Cluster1273|203|SNP|T|17|Male(T/C)

>Cluster1273|203|Indel|T|189|Male(T/-2TG)

1 GgtACAGCTGTTATGACGTCCAGAGGGTTGGTTTTGCAATAATtGCAGAC

51 TTTCTCCTTAGAGCAGCTCAGATTGCGAGTGCAGTACAAAGGGAAAATGG

101 CACGTTGTAGAAACAATTCCTGAAGCCTGAAGGGTCTAAAAACAATATAA

151 GTATTGATGAGCTCCGAAACAAGTTTGAGACGTTTGCAT--TGCCCCGGG

201 GAA

$>$ Cluster48400|221|SNP|C|63|Female (C/C)

>Cluster48400|221|SNP|C|153|Female (C/C)

1 GGTTGCATGCATGCAGAATCAACATCACACAGCAATTGCACAAGTCAATA

51 TAACCCTAGATTCGACAAATAAACTTCATTTTTTTTAATTGAAGACGGGT

101 CATTTGGCAGGATGCAGCTCGAAGCAGGCTCGAGGTAAATGACAATATTA

151 AGCAGTAACTCAGATGTGTAAATGACAGTCAGTAAAAGCCGAAGTACGAA

201 AAGCAGAAATTTACCGGGGAA

$>$ Cluster $48400|221|$ SNP|C|63|Male (C/T)

$>$ Cluster48400|221|SNP|C|153|Male (C/T)

1 GGTTGCATGCATGCAGAATCAACATCACACAGCAATTGCACAAGTCAATA

51 TAACCCTAGATTTGACAAATAAACTTCATTTTTTTTAATTGAAGACGGGT

101 CATTTGGCAGGATGCAGCTCGAAGCAGGCTCGAGGTAAATGACAATATTA

151 AGTAGTAACTCAGATGTGTAAATGACAGTCAGTAAAAGCCGAAGTACGAA

201 AAGCAGAAATTTACCGGGGAA

>Cluster72369|270|SNP|A|57| Female (A/A)

>Cluster72369|270|SNP|G|154|Female (G/G)

1 GCTTATGTTACCAAGCTAGCCAAGGTAATGCCTCCACCAACATTTTCTTT

51 ATATATACACGTCTTAGTTTGATTATTTTTGTACAGAGACTTGACTCCGA

101 CCTTTAATTATTTTATTGGATATTCAAAAGTGTCCTCTGCATTTTCAGCT

151 GTAG---AACTCTTGAACATATTCCTGGTCAAAATTATTCAATTTCATAT

201 GCAATTCAATAGAAAATTACAAAGTTTTTAACTCAATGAGAGAGATTGTG

251 TAGATTACTTTTTATTCCTG

>Cluster72369|270|SNP|A|57| Male (A/T)

>Cluster72369|270|SNP|G|154|Male (G/+3ACC)

1 GCTTATGTTACCAAGCTAGCCAAGGTAATGCCTCCACCAACATTTTCTTT

51 ATATATTCACGTCTTAGTTTGATtATtTTtGTACAGAGACTTGACTCCGA

101 CCTTTAATTATTTTATTGGATATTCAAAAGTGTCCTCTGCATTTTCAGCT

151 GTAGACCAACTCTTGAACATATTCCTGGTCAAAATTATTCAATTTCATAT

201 GCAATTCAATAGAAAATTACAAAGTTTTTAACTCAATGAGAGAGATTGTG

251 TAGATTACTTTTTATTCCTG 